\section{Asthmastudie: Alles unter Kontrolle? Keine Spur!}

\begin{abstract}
In einer mit zwei verschiedenen Placebomaßnahmen kontrollierten
Studie erlebten Asthmapatienten unter aktivem Salbutamol, Schein-Salbutamol und Scheinakupunktur eine gleich ausgeprägte Verbesserung ihrer Symptomatik, obgleich die $\mathrm{FEV}_{1}$ nur unter aktivem Salbutamol zunahm.
\end{abstract}

$\mathrm{n}$ prospektiven experimentellen Studien bei Asthmapatienten ist es schwierig zu entscheiden, ob das Ansprechen auf Placebomaßnahmen sich vom sehr variablen natürlichen Krankheitsverlauf unterscheidet. In einer doppelblind geführten Pilotstudie unterzog man 46 Asthmapatienten vier verschiedenen Maßnahmen: einer Inhalation von Salbutamol, einer Placeboinhalation, einer Scheinakupunktur und keiner Intervention. Die Maßnahme „keine Intervention" schien eher dazu angetan, die Patienten zu verärgern: Man bestellte sie ein, ließ sie mehrere Stunden warten und schickte sie dann wieder nach Hause.

Die vier Maßnahmen wurden in einem Blockdesign in randomisierter Reihenfolge durchgeführt, wobei die Zeitabstände zwischen den Konsultationen 3-7 Tage betrugen. Bei jedem Be-

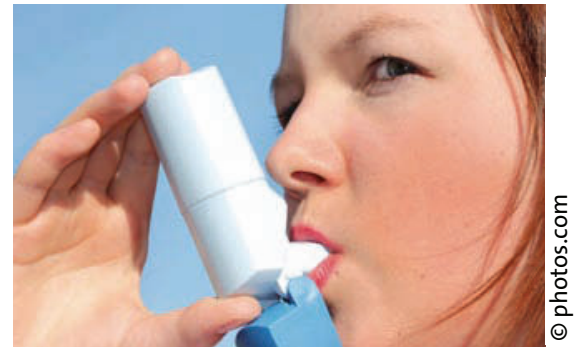

Paradox: FEV1 unverändert, aber die Patientin fühlt sich besser.

such wurden über zwei Stunden hinweg Spirometrien durchgeführt, die $\mathrm{FEV}_{1}$ bestimmt und die vom Patienten empfundene Erleichterung der Symptomatik anhand eines standardisierten Protokolls dokumentiert. Immerhin 39 Patienten beendeten die Studie wie vorgesehen.

Die Inhalation von Salbutamol führte erwartungsgemäß zu einer 20\%- igen Zunahme der $\mathrm{FEV}_{1}$, während sie unter den drei übrigen Interventionen nur um $7 \%$ zunahm. Zur Überraschung der Forscher korrelierte dieser Befund allerdings nicht mit dem subjektiven Eindruck der Patienten. Ihre empfundene Verbesserung lag nach VerumSalbutamol bei $50 \%$, jedoch auch nach der Placeboinhalation und der Scheinakupunktur bei 45 bzw. 46\%. Lediglich nach der Nichtintervention unterschied sich der subjektive Eindruck signifikant von den drei übrigen Maßnahmen, wobei es allerdings auch dabei noch zu einer Verbesserung von 2\% kam.

Fazit: Die Patienten empfanden nach den Placebomaßnahmen eine Besserung ihrer Asthmasymptomatik, obwohl das laut den $\mathrm{FEV}_{1}$-Werten nicht hätte sein dürfen. Die Studienautoren schlussfolgern, die Berichte der Patienten seien „unzuverlässig“. Dabei ignorieren sie jedoch die Tatsache, dass Krankheitsgefühl und Leidensdruck komplexe Vorgänge sind, die nicht mit einer schlichten $\mathrm{FEV}_{1-}$ Messung abgebildet werden können.

Prof. Dr. H.-S. Füessl

Wechsler ME et al. Active albuterol or placebo, sham acupuncture, or no intervention in asthma. New Engl J Med 2011;365: 119-26

\title{
Liposomen gegen Heuschnupfen?
}

\section{Bei der Entstehung allergischer Erkrankungen spielen Störungen der Mukosabarriere eine wichtige Rolle. Kann eine Stabilisierung der nasalen Mukosabarriere mittels Liposomen die Symptome einer saisonalen allergischen Rhinokonjunktivitis lindern?}

l: n der kontrollierten prospektiven offenen Studie wurde die Wirkung von Liposomen mit der von Cromoglycat verglichen. 72 Patienten mit einer saisonalen allergischen Rhinokonjunktivitis (SAR) wurden abhängig von ihren Symptomen und Präferenzen einer von drei Therapiegruppen zugeordnet: einem Liposomen-Nasenspray (LipoNasal $\mathrm{n}$ ) in Monotherapie, einer Kombination aus Liposomen-Nasenspray und -Augenspray (TearsAgain) oder einer Cromoglycat-Kombinationstherapie. Sieben Tage lang dokumentierten die Patienten na- sale und konjunktivale Symptome anhand einer diskreten Vier-Punkte-Skala ( 0 = keine, 3 = starke Beschwerden).

Unter allen Therapien wurde ein schneller Wirkungseintritt beschrieben. Gut zwei Drittel der Patienten bemerkten schon in der ersten Stunde eine Besserung. Mit dem Liposomen-Nasenspray und mit Cromoglycat besserten sich Nasen- und Augensymptome signifikant und in vergleichbarem Maß. Mit der Liposomenkombination war der Effekt nur in Bezug auf die Rhinitis signifikant. In allen drei Gruppen lag der
Effekt über der minimalen klinisch relevanten Differenz (MCID). Alle Therapien wurden gut vertragen, Nebenwirkungen wurden nicht beobachtet.

Fazit: Die Studienautoren sehen in den Liposomen-Präparaten eine ,viel versprechende Alternative" für die Behandlung von SAR-Patienten, insbesondere wenn die Patienten eine pharmakologische Behandlung ablehnen. Als möglichen Wirkmechanismus der Liposomen vermuten sie eine Stabilisierung der nasalen Mukosabarriere, die den Eintritt von Allergenen erschwert.

Dr. Beate Schumacher

Böhm M et al. Liposomes: a new nonpharmacological therapy concept for seasonal-allergic-rhinoconjunctivitis. Eur Arch Otorhinolaryngol 2011; 15. Jul [Epub ahead of print] 